\title{
Research on the control strategy of phase balance in H-bridge cascade SVG
}

\author{
Anfei $\mathrm{Xu}{ }^{1}, *$ Linbo $\mathrm{Gan}^{2}$, Ziqiang $\mathrm{Xi}^{3}$ and Xiaogang $\mathrm{Du}^{4}$ \\ Hubei Key Laboratory of Solar Energy Efficient Utilization and Energy Storage System Operation Control, Hubei University of \\ Technology, Wuhan 430068
}

\begin{abstract}
Based on the H-bridge cascaded SVG as one of the best solutions to effectively compensate reactive currents, this article focuses on the cascaded high-voltage H-bridge SVG phase balance problem. While analyzing the influence factors of the SVG DC side voltage, it summarizes and introduces 5 SVG phase balance control strategy, Analyzed its real-time performance, stability, applicable occasions, algorithm and hardware circuit complexity, etc.Some control strategies have been simulated and verified, and the simulation results have reached the expected value, which provides a reference for the selection of balance control strategies for high-voltage SVG equipment.
\end{abstract}

\section{Introduction}

With the development of power electronic technology and the widespread use of power electronic equipment, the harmonics and reactive power loss of the power grid have been greatly increased,and the safety and stability of the power grid have been greatly affected.Therefore, the compensation of reactive power has become a hot spot of current research.Static var generator (SVG) came out at the historic moment.Because of its good compensation effect and fast response speed, it has been widely used in improving power quality and compensating reactive power[1-5].

As a new type of inverter device in the power electronics industry that can compensate reactive power efficiently and in real time,SVG has been widely used in various low-voltage Medium and high pressure field.However,the fluctuation of the DC side capacitor voltage will eventually deviate from the set value due to the loss of the switching devices, the pulse delay, the electromagnetic influence between the components and the difference of the circuit device parameters between the H-bridge inverter units. Will directly affect the compensation effect of the device and the quality of the output waveform[6-13], when it's serious, It may even cause the voltage on the switching device to exceed the withstand voltage level,causing the device to burn. Therefore, the research on the DC side voltage control method of H-bridge cascaded SVG has become a hot topic and a key problem for scholars at home and abroad[14-21].

This paper analyzes and studies the voltage balance control strategies between the sub-modules in the five cascaded SVG, and compares them in terms of real-time performance, stability, hardware circuits, and control algorithms, and summarizes the applicable occasions for each control strategy.The selection of high-voltage SVG equipment balance control provides reference.

\section{Analysis on the influence factors of DC side capacitor voltage}

Since the structure of each sub-module of cascaded SVG is exactly the same, and in normal operation, the DC side capacitance of each sub-module is independent of each other, and the trigger pulse of each sub-module is exactly the same, so the DC side capacitor voltage will always be maintained in a balanced state.But this is not the case,due to uncontrollable factors such as the loss of switching devices, pulse delay, and electromagnetic influence between the components in each units, the system cannot always be in a stable state,even in an ideal state. Then the structural parameters of the sub-module H-bridge circuit cannot be exactly the same, resulting in the imbalance of the internal sub-module capacitor voltage of each phase.Now we use a separate sub-module for voltage balance analysis, as Figure 1.

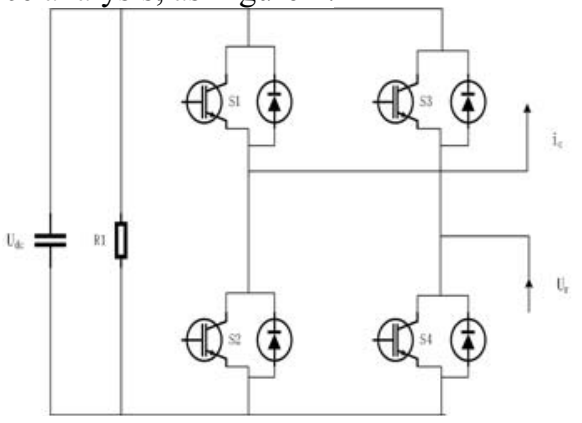

(a)H-bridge individual sub-module circuit diagram

\footnotetext{
*Corresponding author: 244318483@qq.com
} 


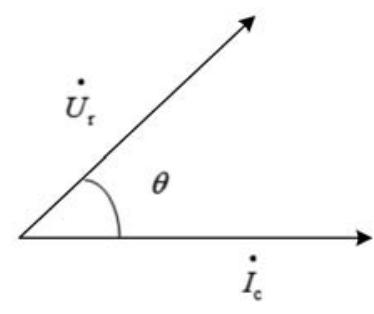

(b) H-bridge separate sub-module vector Fig. 1. Analysis diagram of $\mathrm{H}$ bridge individual sub-module

Figure 1 shows the circuit diagram and vector diagram of the H-bridge sub-module,In figure 1(a) $U_{d c}$ is the Sub-module unit DC side voltage, $U_{r}$ is the output voltage, $i_{c}$ is the output phase current, R1 is the equivalent resistance of capacitance loss, $\Theta$ is the vector angle between output voltage and current in figure 1(b), from the power balance, the capacitance energy of the DC side of the sub-module can be changed as :

$$
\Delta P_{\mathrm{c}}=P_{1}-P_{2}
$$

$P_{1}$ is the absorbed active power, $P_{2}$ is the active power lost by the sub-module itself; and

$$
\begin{aligned}
& P_{1}=U_{\mathrm{r}} I_{\mathrm{c}} \cos \theta \\
& P_{2}=P_{3}+P_{4}
\end{aligned}
$$

$P_{3}$ is the equivalent resistance loss; $P_{4}$ is the mixed loss, which mainly considers the switching loss of the components, so:

$$
\begin{gathered}
P_{3}=\frac{U_{d c}^{2}}{R} \\
P_{4}=\frac{4}{\pi} f\left[E_{o n}(\text { nom })+E_{o f f}(\text { nom })\right] \frac{I_{c} U_{d c}}{I_{\text {nom }} U_{\text {nom }}}
\end{gathered}
$$

$I_{\text {nom }}$ and $U_{\text {nom }}$ are the rated current and rated voltage of the switching device, $E_{\text {on }}$ (nom) and $E_{\text {off }}$

(nom) are the the turn-on and turn-off losses of the switching device at rated voltage and rated current.Combining the above formulas, the energy change of a single sub-module is:

$$
\Delta P_{\mathrm{c}}=U_{\mathrm{r}} I_{\mathrm{c}} \cos \theta-\frac{U_{d c}^{2}}{R}-\frac{4}{\pi} f\left[E_{\text {on }}(\text { nom })+E_{\text {off }}(\text { nom })\right] \frac{I_{c} U_{d c}}{I_{\text {nom }} U_{\text {nom }}}
$$

From equation(6), The imbalance factor that causes the sub-module's own energy change is mainly caused by $U_{r}, \theta, R, E_{\text {on }}$ (nom) and $E_{\text {off }}$

(nom) by these parameters, Theoretically,by changing these factors, the voltage balance between the sub-modules in the phase can be achieved, but in actual operation, $E_{\text {on }}($ nom $)$ and Eoff (nom) of each sub-module itself cannot be consistent,so it can only adjust $P_{1}$, which is the active power absorbed by itself[22].

\section{The method of DC side phase balance method}

\subsection{Sub-unit micro-control}

After comparing the actual value of the DC side capacitor voltage in each phase with the average value, it is adjusted by the PI controller, and the obtained value is multiplied by the current of each phase H-bridge unit module to obtain the fine-tuning command of each phase modulation wave. The fine-tuning command is added to the modulation wave obtained by the SPWM modulation strategy to obtain the actually applied modulation wave, which can reasonably allocate the active power input by each power unit to maintain the DC side capacitor voltage balance[23]. The control chart is shown in Figure 2 .

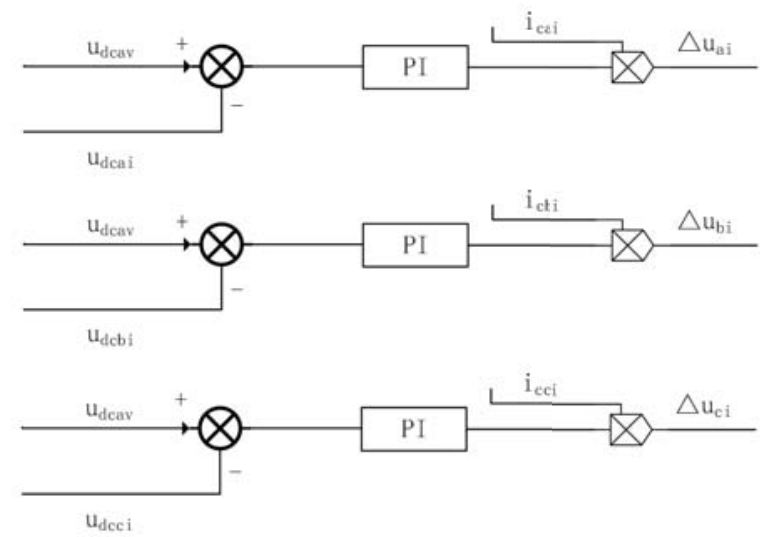

Fig. 2. Sub-unit micro-control control block diagram

$U_{d c s i}$ is the actual voltage value of the $\mathrm{i}$-th sub-module on phase $\mathrm{S}, u_{d c s v}$ is the average voltage on phase $\mathrm{S}, i_{c s i}$ is the current value of the $\mathrm{i}$-th sub-module on phase $\mathrm{S}, \Delta \mathrm{u}_{\mathrm{si}}$ is the fine-tuning instruction obtained by the i-th sub-module of the $\mathrm{S}$ phase[24].

The advantage of this method is to precisely control the active power of each sub-module, it can keep the same voltage for each sub-unit of each phase,so as to maintain in phase balance,the disadvantage is that when the number of sub-modules is large, if a certain group of devices fails and is not detected,it will affect the value of the phase voltage, resulting in deviations in the entire voltage balance system.Secondly, errors may occur in detection speed and signal processing, and the real-time performance is not ideal.So this method is only suitable for systems with a small number of sub-modules.

\subsection{Sub-module fine-tuning}

$\mathrm{N}-1$ modules per phase, according to the DC side voltage of the module, use the square of the average value of the DC side voltage of each series-connected module of phase $\mathrm{A}$ as the command,and use the square of the actual DC side voltage of each module as the feedback,through PI regulator adjustment, the output current is normalized, and then multiplied by the A-phase output current per unit value, then the fine-tuning amount of the modulation wave of the A-phase corresponding module is obtained. 
Add the fine-tuning command and the original command voltage as the final command voltage of each H-bridge module of phase A of the H-bridge cascaded SVG. Each module N-1 fine-tunes its command voltage along the output current direction of the converter,adjusts the active power it absorbs,and achieves the purpose of controlling the DC side voltage balance of each module. As shown in Figure 3.

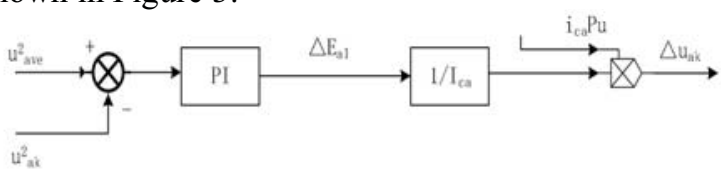

Fig. 3. Sub-module fine-tuning control block diagram

$u^{2}$ ave is the square of the average value of phase A voltage, $\mathrm{u}^{2}$ ak is the square of the actual voltage of the k-th sub-module of phase A, $\Delta E_{a l}$ is the AC side command voltage PI regulator adjustment amount of the module, $I_{c a}$ is A phase output current[25].

The design of PI controller parameters, as shown in Figure 4.

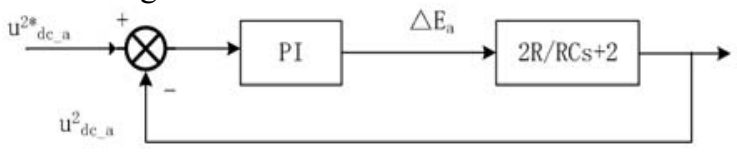

Fig. 4. Block diagram of single-phase DC side voltage PI controller parameter design

This method has high accuracy and feasibility, and also gives the design method of PI controller parameters, which is suitable for a variety of high-voltage SVG systems. Its advantages are high real-time performance, relatively easy calculations, and excellent dynamics.It has become one of the commonly used methods at present, but in some unbalanced scenarios, this method has a slight defect, so it also has limitations in application.

\subsection{Triangular Wave Comparison}

This method first feeds back the SVG output current, clarifies the input voltage and feedback value, and then inputs the signal to the PI controller. After analysis and processing, and then compares the triangle wave, a certain pulse signal will be formed, The output signal value controls the PWM output signal, thereby controlling the conduction state of the internal components of the main circuit, and finally achieving phase balance[26].In the practical application stage, the method is considered from the $\mathrm{d}-\mathrm{q}$ axis, and the basic principle is shown in Figure 5.

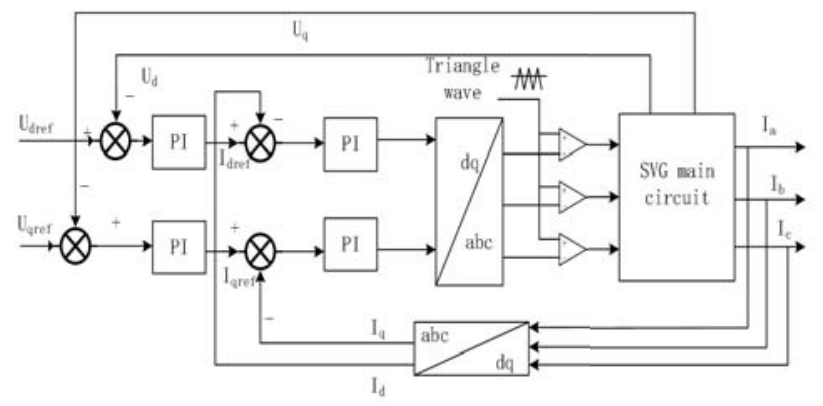

Fig. 5. Control block diagram of triangle wave comparison method on d-q axis

$U_{d}$ and $U_{q}$ are respectively the output feedback voltage on the dq axis, $U_{\text {dref }}$ and $U_{\text {qref }}$ are reference voltage in the SVG DC.Let $U_{d}$ and $U_{d r e f}, U_{q}$ and $U_{q r e f}$ subtract first,get the difference and input it into the PI regulator. When it reaches this module, the output $I_{\text {qref }}$ and $I_{d r e f}$ is the active current formed by the direct current in the system.Secondly, invert the SVG, output three-phase AC values, namely $I_{a}, I_{b}$, and $I_{c}$ for Parker transformation, and make them as the value feedback values $I_{d}$ and $I_{q}$. Subtract the two values from $I_{\text {dref }}$ and $I_{\text {qref }}$ again to obtain two results, then it is sent to the PI module, the output value depends on the Parker inverse transformation to determine the three-phase value,and then compares and analyzes with the triangle wave, then the PWM waveform can be output to control the conduction state of the sub-modules in the main circuit, thereby achieving full system control[27].

This method uses instantaneous values to calculate, and its advantage is that the response is relatively fast; it compares DC circuits and has high stability. The disadvantage is that the hardware circuit is too complex, the operating speed is low, and the error is large. The most important point is that this method has strict standards for the switching frequency, so the durability of the switch must be paid enough attention. It is common in small and medium capacities, and the sensitivity is not strict. Limitations, low measurement cost, and small component replacement loss in the SVG system.

\subsection{Phase amplitude method}

When the phase of the output voltage of the SVG sub-module with respect to the output current of the SVG is not equal to $90^{\circ}$, the total charging energy of the capacitor voltage will not be 0 . Correspondingly, the increase or decrease of the capacitor voltage will directly lead to the failure of the DC voltage of the H-bridge sub-module balance.Therefore, the DC voltage of each H-bridge sub-module must be individually controlled. That is, by correcting the phase difference between the output voltage and current of each sub-module and the output voltage amplitude to ensure that the DC side voltage of each sub-module is equal and equal to the average value of the DC voltage of each phase, so as to solve the voltage imbalance in the SVG problem. The control block diagram is shown in Figure 6 . 

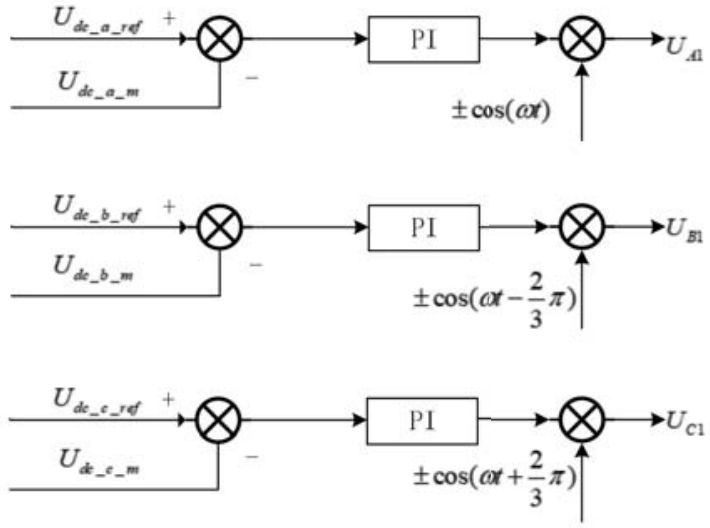

Fig. 6. Control block diagram of phase amplitude method

Cosøt explain that the phase difference between $\mathrm{U}_{\mathrm{A}}$ and the system voltage is $90^{\circ}$. By adjusting the amplitude of $U_{A 1}$ and the phase relationship with the output current of SVG, the active power can be absorbed or released to achieve the purpose of balancing the DC voltage of each sub-module[28].

This method is to ensure that the DC side voltage of each sub-module is equal by controlling the phase difference between the output voltage and current of each sub-module and the amplitude of the output voltage. The hardware circuit is relatively simple, with high stability, dynamic characteristics, etc.

\subsection{Adaptive PI control method}

\subsubsection{Principle of adaptive PI control method}

In the entire voltage balance control system, a large number of PI controllers are needed. In order to overcome the defects of slow response speed, poor real-time performance, and poor tracking performance of SVG devices due to the fixed PI parameters.A method based on adaptive PI control to replace traditional PI control is proposed. This method can use PI adjustment parameters to adaptively identify and control errors on-line under the condition of load jumps until the controller reaches the maximum In good condition[29].

The adaptive PI algorithm is as follows:

$$
\begin{gathered}
K_{L}=\frac{\Delta i}{\Delta t} \\
L=K_{1} K_{L} \\
R=\frac{u(t)}{i(t)} \\
K_{P}=\frac{K_{2} L Y^{*}}{R X\left(Y^{*}-Y\right)} \\
K_{I}=K_{3} K_{P}-K_{4} L R
\end{gathered}
$$

In the formula, $K_{L}$ is the inductance calculation coefficient, $K_{1}, K_{2}, K_{3}$ and $K_{4}$ are the correction coefficients, $L$ and $R$ are the equivalent inductance and resistance values, $\mathrm{X}$ is the controller input value, $Y$ is the controller output value, and $Y^{*}$ is the expected value of controller , $K_{P}$ and $K_{I}$ are the proportional and integral coefficients of the adaptive PI regulator, respectively. Use the correction coefficient to continuously update the relevant parameters to complete the entire adaptive PI control process.

In terms of the voltage balance of the sub-modules in the phase, we superimpose a fine adjustment amount on the basis of the main modulation wave to adjust the size of the modulation wave to achieve the purpose of controlling the DC side voltage.

Take the ratio of the instantaneous compensation current $\mathrm{i} 0$ of the SVG to the maximum compensation current $\mathrm{I} 0$ in a period as a certain AC signal $\hat{\mathrm{S}}$;According to the actual voltage value and reference value of each unit, the modulation wave modulation amount can be obtained $\Delta V d c i$, then using the opposite of the sum of the first two compensation quantities as the final modulating wave compensation quantity can improve the adaptability when the load changes suddenly[30].

$$
\begin{gathered}
\tilde{S}=i_{0} / I_{0} \\
\Delta V_{d c i}=\lambda\left(u_{d c i}-u_{d c i}^{*}\right)\left(K_{P}+\frac{K_{I}}{s}\right) \tilde{S}
\end{gathered}
$$

In the formula, the positive $d$ negative of $\lambda$ represents the direction of the signal $\hat{\mathrm{S}}$, when $\lambda=1, \mathrm{i}_{0}$ is capacitive; when $\lambda=-1, i_{0}$ is inductive, taking phase $\mathrm{A}$ as an example, its control block diagram is shown in Figure 7.

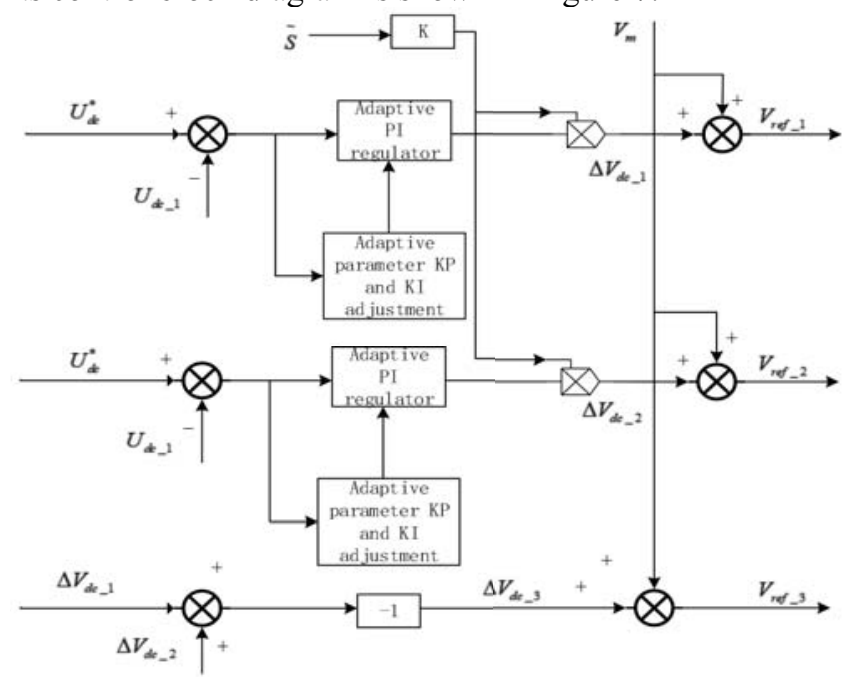

Fig. 7. Control block diagram of adaptive PI control method

This method replaces the traditional PI regulator with adaptive PI, and superimposes a fine-tuning amount of the modulation wave on each sub-module of SVG to control the main modulation wave and the voltage of each sub-module to achieve the purpose of voltage balance control in the phase.The highlights speak for themselves,Compared with traditional PI regulators, the adaptive PI regulator has faster response speed and stronger real-time performance, improves the accuracy of current tracking compensation, and can self-regulate under sudden load changes to achieve the best state. The disadvantage is that the adaptive PI controller is more complicated and the parameters are difficult to set. 


\subsubsection{Simulation}

In order to verify the applicability and feasibility of this method, it is simulated and verified by a simulation platform based on MATLAB/SIMULINK.The main simulation parameters are set as follows:The system line voltage is $3 \mathrm{kV}$, the cascaded $\mathrm{SVG}$ capacity is $300 \mathrm{kVA}$, the DC voltage of each unit is $800 \mathrm{~V}$, and the DC capacitor is $2 \mathrm{mF}$. The main inductance is $3 \mathrm{mH}$, and the switching frequency is 10HZ.In the A phase, three cell voltage simulation graphs are shown in Figure 8.
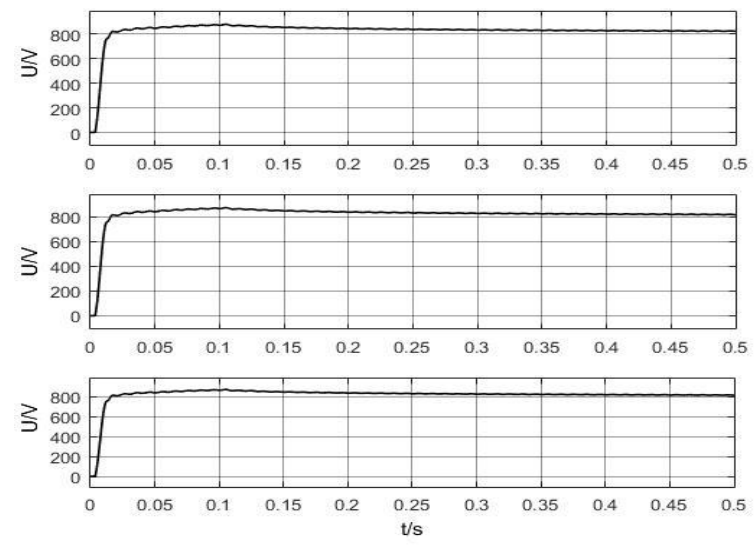

Fig. 8. Voltage waveform of each unit of phase A

It can be seen from the figure that the voltage of each unit rises rapidly from the initial $0 \mathrm{~V}$ voltage and converges to about $800 \mathrm{~V}$ within $0.25 \mathrm{~s}$, and gradually stabilizes, the fluctuation does not exceed $20 \mathrm{~V}$, and the deviation is $2.5 \%$, The pressure of each unit is well realized.The simulation results achieved the balance of capacitor voltage, verified the effectiveness of the algorithm and the compensation performance of the control strategy, and proved that the adaptive PI control method has a faster response speed and better stability in the case of a sudden voltage change.

\section{Analysis}

The prerequisite for the stable operation of the H-bridge cascaded SVG is that the voltages of the DC side capacitors are balanced, and the most difficult point is how to better solve the phase balance without affecting the balance of the entire system.After studying a large number of Chinese and foreign documents, it is found that the current research trend is to directly control the sub-modules, the purpose is to improve the real-time performance, stability and dynamic characteristics of the system.Form 1 summarizes and summarizes the above several methods of SVG phase internal balance control.
Table 1.Comparison of different control methods

\begin{tabular}{|l|l|l|l|}
\hline method & real-time & stability & $\begin{array}{l}\text { Hardware } \\
\text { circuit }\end{array}$ \\
\hline $\begin{array}{l}\text { Sub-unit } \\
\text { micro-control }\end{array}$ & moderate & lower & simpler \\
\hline $\begin{array}{l}\text { Sub-unit } \\
\text { fine-tuning }\end{array}$ & higher & higher & moderate \\
\hline $\begin{array}{l}\text { Triangular } \\
\text { Wave } \\
\text { Comparison }\end{array}$ & high & high & $\begin{array}{l}\text { more } \\
\text { complicated }\end{array}$ \\
\hline $\begin{array}{l}\text { Phase } \\
\text { amplitude } \\
\text { method }\end{array}$ & higher & higher & moderate \\
\hline $\begin{array}{l}\text { Adaptive PI } \\
\text { control } \\
\text { method }\end{array}$ & high & high & complicate \\
\hline
\end{tabular}

From the analysis of the above table, we can see that there are many methods for phase voltage balance at home and abroad. The most difficult point is how to efficiently solve the problem of phase voltage imbalance without affecting the balance of the entire system. The above-mentioned methods all have their adaptable SVG systems. Nowadays, a more widely used method is the sub-unit fine-tuning method, but in the case of voltage sudden changes, the control effect of this method is somewhat deviated.Therefore, how to perform effective intra-phase voltage balance control under unstable or abrupt voltages has become the focus of future phase intra-phase balance research.

\section{Conclusion}

The application of SVG technology has greatly promoted the research and development of the DC side voltage balance control strategy, which is helpful to improve the stability and real-time performance of the system, etc., and greatly reduces the loss of components and the hidden danger of the system. This paper introduces the sub-unit micro-control method, the sub-unit fine-tuning method, the triangle wave comparison method, the phase amplitude method and the adaptive PI control method by consulting a large number of documents, and compares and analyzes their various characteristics and adaptation occasions.Among them, the adaptive PI control method was simulated and verified. The simulation results achieved a good balance of capacitor voltage and verified the effectiveness of the algorithm and the compensation of the control strategy. It provides a higher reference value for the selection of high-voltage SVG equipment balance control strategy.

\section{References}

1. L.yungui,H. lijun,L. guojie.A Circulating Current Suppression Method for MMC-based Rectification System with Asymmetric Bridge Arms [J].Electric Automation, 2017,39 (02):12-15. 
2. Akagi H. Classification, terminology, and application of the modular multilevel cascade converter ( MMCC ) $[\mathrm{J}]$. IEEE Transactions on Power Electronics , 2011, 26 (11): 3119-3130.

3. Z. Bo,G. jianbo, Z. fei. DC Voltage Balance Control Strategy Among Phases for Cascaded STATCOM $[\mathrm{J}]$. Proceedings of the CSEE, 2012, 32 (34) : 36-41.

4. Y. xiaodong, W. conglin, S. liping , etc. Generalized Space Vector PWM Method for Cascaded Multilevel Inverters [J].Transactions of China Electrotechnical Society, 2014, 29 (6) :145-152.

5. W. baoan,S. jiao,C. hao.Phase-current reference calculation for SVG used in comprehensive management of single-phase load power quality [J].Electric Power Automation Equipment,2016,36(02):57-64.

6. L.yunfeng, H. yingjie, Y. shiqi, W. yue. L. jinjun .Phase-current reference calculation for SVG used in comprehensive management of single-phase load power quality [J].Transactions of China Electrotechnical Society,2015,30(05):23-32.

7. L. Zhao, L. bangying, D. shanxu, etc. Research on Cascade Multilevel STATCOM Under Unbalanced System Voltage [ J $]$. Proceedings of the CSEE, 2011, 31(9): 1-7.

8. W. cong, W. chang, J. jian,etc . New Cascade Diode H-Bridge Multi-Level Rectifier $[\mathrm{J}]$. Transactions of China Electrotechnical Society, 2014, 29(1) : 273-281.

9. W. shunliang, S. wensheng, F.xiaoyun. One fast voltage-balancing method of single phase cascaded H-bridge rectifiers[J].Electric Machines and Control,2016,20(05):37-44.

10. L.lingling, L. xiuxue, J. haitao, L. zhigang.A Balancing Control Method of DC Side Voltage for Cascaded H-Bridge Static Var Generator[J].Transactions of China Electrotechnical Society,2016,31(09):1-7.

11. G. juncheng, L.wenhua ,Y. zhichang .RESEARCH ON THE VOLTAGE UNBALANCE OF DC CAPACITORS OF CASCADE STATCOM Part One Simulations and Experiments[J].Automation of Electric Power Systems,2003(16):53-57+86.

12. X. Huiyong. Reactive Power Compensation in SVG Technology Research Present Situation and the Development. Applied Energy Technology, 2012(2): 31-33.

13. L. wenya, Y. gang ,H. ruan, L.kai ,Z. lidan Research of DC voltage balance control based on multi-level cascaded APF $[J]$.Power System Protection and Control,2015,43(04):94-101.
14. PENG F Z,WANG J.A universal STATCOM with delta - connected cascade multilevel inverter [C] / / IEEE Power Electronics Specialists Conference,2004,( 35):3529-3533.

15. B. Mumyakma, XiaHe Jin, ChangChang Wang. Static Var Com-pensator with Neural Network Control[C]. Transmission and Distribution Conference, IEEE, 2012: 542-549.

16. L. Jianlin, Z. dongli, Z. bing, etc. Caseade H-bridge Coventer with Carrier Phase Shifted Technique and Its Application in Active Power Filter [ $\mathrm{J}]$. Proceedings of the CSEE,2006,26(10) :109-113.

17. W. zhaoan, Y. jun, L. jinjun. Harmonic Suppression and Reactive Power Compensation Technology[M] .Mechanical Industry Press, 1998.

18. D. ming, C. zhong, Z. guorong, etc.Passive and active schemes of DC ripple suppression for cascaded H-bridge converter of energy storage system [ J $]$.Electric Power Automation Equipment,2016,36 (4):19-24.

19. Chou S F, Wang B S, Chen S W, et al. Average power balancing control of a STATCOM based on the cascaded H-bridge PWM converter with star configuration $[\mathrm{J}]$. IEEE Transactions on Power Electronics, 2013, 50(6) : 3893-3901.

20. Yang X W, Jiang J G, Liu S C. A novel design approach of dc voltage controller for cascaded H-bridge converter-based STATCOM [C] / / Power Electronics and Motion Control Conference , Wuhan, China, 2009: 2359-2364.

21. Vazquez S, Leon J I, Carrasco J M, et al. Analysis of the power balance in the cells of a multilevel cascaded H-bridge converter[J]. IEEE Translations on Power Electronics, 2010, 57( 7) : 2287-2296.

22. W. kexin.Control Strategy Research and Equipment Development of High Voltage Cascaded Static Var Generator[D].Hunan University,2016.

23. T .mingxing, Y. hong, Z.yuxin.A Balancing Control Method of DC Capacitor Voltage for Cascaded H-Bridge SVG[J].Power $\quad$ System Technology,2013,37(09):2632-2638.

24. L. xuqiang, Z. zeying, A. xutao , C. zhiqiang. Research on Control Strategy for Cascade H-bridge SVG[J].Electrical Automation, 2019,41(02):4-7.

25. H. yingjie, F. yabing, D. wenyan. Research on DC Voltage Balancing Control Method of Star Connection Cascaded H Bridge Static Var Generator [J].Transactions of China Electrotechnical Society,2016,31(11):13-21.

26. B.K.Keshavan and Nagesh Prabhu.Damping of Subsynchronous Oscillations Using STATCOM-A FACTS Controller. 2004 International Conference on Power System Technology, 2014.

27. G. sance. Design and Implementation of SVG Reactive Power Compensation Device[D].Northeast Agricultural University,2019. 
28. L. guiying, D. mingfeng, S. mingshi, M. zhiyong, Z. bingyu, L. mei Novel DC Voltage Control Method of $\mathrm{H}$ Bridge Cascaded STATCOM[J].Proceedings of the CSU-EPSA,2015,27(10):48-55+68.

29. LIU Z, LIU B Y, DUAN S X, et al. A novel DC capacitor voltage balance control method for cascade multilevel STATCOM $\quad[\mathrm{J}]$. IEEE Transaction on Power Electronics, 2012, 27 ( 1) : 14 $-27$.

30. P. yonglong, $H$. jianghao, L. yabing, D. peng, $H$. ning.DC side voltage balancing control for medium voltage cascaded $\mathrm{H}$ bridge SVG[J].Electric Power Science and Engineering,2018,34(06):1-8 